\title{
Local discontinuous Galerkin method for distributed-order time-fractional diffusion-wave equation: Application of Laplace transform
}

\author{
Hadi Mohammadi Firouzjaei ${ }^{1}$, Hojatollah Adibi ${ }^{1}$, and Mehdi Dehghan ${ }^{2}$ \\ ${ }^{1}$ Amirkabir University of Technology \\ ${ }^{2}$ Amirkabir University of Technology,
}

May 5, 2020

\begin{abstract}
In this paper, the Laplace transform combined with the local discontinuous Galerkin method is used for distributed-order time-fractional diffusion-wave equation. In this method,at first, we convert the equation to some time-independent problems by Laplace transform. Then we can solve these stationary equations by the local discontinuous Galerkin method to discretize diffusion operators at the same time. Then, by using a numerical inversion of the Laplace transform we can find the solutions of the original equation. One of the advantages of this procedure is its parallel implementation. Another advantage of this approach is that the number of stationary problems that should be solved is much less than that are needed in time-marching methods. Finally, some numerical experiments have been provided to show the accuracy and efficiency of the method.
\end{abstract}

\section{Hosted file}

wileyNJD-AMA.pdf available at https://authorea.com/users/300850/articles/430577-localdiscontinuous-galerkin-method-for-distributed-order-time-fractional-diffusion-waveequation-application-of-laplace-transform

Hosted file

Figures.rar available at https://authorea.com/users/300850/articles/430577-localdiscontinuous-galerkin-method-for-distributed-order-time-fractional-diffusion-waveequation-application-of-laplace-transform 